Preprint for: Olkoniemi, H., \& Kaakinen, J. K. (2021). Processing of irony in text: A systematic review of eye-tracking studies. Canadian Journal of Experimental Psychology/Revue canadienne de psychologie expérimentale. Advance online publication. https://doi.org/10.1037/cep0000216

(C) 2020, Canadian Psychological Association. This paper is not the copy of record and may not exactly replicate the final, authoritative version of the article. Please do not copy or cite without authors' permission. The final article will be available, upon publication, via its DOI: 10.1037/cep0000216

\title{
Processing of irony in text: A systematic review of eye-tracking studies
}

\author{
Henri Olkoniemi \& Johanna K. Kaakinen
}

University of Turku

\section{Author Note}

This research was supported by a grant from the Ella and Georg Ehrnrooth Foundation awarded to Henri Olkoniemi. Data and analysis code for this study have been made available at https://osf.io/w5fbs/.

Correspondence concerning this article should be addressed to Henri Olkoniemi, Department of Psychology, FI-20014 University of Turku, Finland. Email: henri.olkoniemi@utu.fi 


\begin{abstract}
Theoretical models of irony comprehension pose different hypotheses about the time-course of resolving ironic interpretation of an utterance, and propose several context-, phrase- and reader-related factors that influence the ease or difficulty of processing irony. In recent years, these factors have been examined using eye tracking, which allows a detailed analysis of time-course of reading processes. In this paper, we present a meta-analysis of the eye tracking studies on irony, and then present a systematic review of the factors that have been shown to influence the time-course of irony processing. The review will point to future directions in how eye tracking could best be applied to further develop the current theoretical views.
\end{abstract}

Keywords: irony, figurative language, eye movements

\title{
Public significance statement
}

Understanding ironic criticism is often hard and requires more time than understanding literal phrases. Recent research suggests that some phrases are harder to comprehend as ironic than others, and the context in which the ironic phrase is used as well as readers' cognitive and emotional capacities play a significant role in how easy it is to resolve its meaning. Current theories of irony comprehension should be developed further to accommodate the findings from these recent studies. 


\section{Processing of irony in text: A systematic review of eye tracking studies}

Written irony can be defined as the use of words to express something other than, and especially intending the opposite of, the literal meaning (Attardo, 2000). The most typical form of irony is ironic criticism, in which something positive is said to indicate a negative meaning (e.g., Kreuz \& Link, 2002). For example, if it is raining heavily and your friend sends you a text message "What a lovely weather for a picnic!" your friend probably means that the weather is not nice. Using ironic criticism is risky because it is easily misunderstood (Hancock, 2004), which is why it is important to understand the processes and factors underlying comprehension of irony. The purpose of the present study was to 1) examine how ironic criticism is processed and comprehended, and 2) identify factors that impact the ease (or difficulty) of understanding ironic criticism. To this end, we conducted a metaanalytic and systematic review of previous eye movement studies, which provided detailed information about the time-course of processes underlying comprehension of written irony.

Different theoretical views emphasize the importance of either context-, phrase- or readerrelated factors in irony comprehension. The classical standard pragmatic view (Grice, 1975) posits that the discrepancy between the literal meaning of the phrase and the discourse context is central to irony processing. First, a reader makes a literal interpretation of the phrase. Second, a discrepancy between the literal interpretation and the context is detected. Finally, the reader seeks for an alternative interpretation and comprehends that the statement is irony. According to this view, comprehending irony should be more difficult and take more time than comprehending a literal statement. Another view emphasizing the importance of context is the direct access view (Gibbs, 1994), which states that literal meaning is not necessarily accessed first, and that a reader may sometimes access the ironic meaning directly. This means that in irony-favorable circumstances processing of ironic statements might be as fast as or even faster than processing of literal statements. This view is supported by 
research showing that the discourse context and cultural expectations that arise in it may influence comprehension of irony (Katz, Blasko, \& Kazmerski, 2004), and repeated exposure to irony might sensitize readers to it (Spotorno \& Noveck, 2014). In addition, one factor that makes comprehending irony difficult is that it conveys an emotional message that differs from the meaning of the literal interpretation (e.g., Toplak \& Katz, 2000), which emphasizes the importance of the discourse context. For example, even though ironic criticism might be perceived as insulting, ironic comments are also used as a form of humor and can be funny. Similarly, the echoic mention theory (Sperber \& Wilson, 1981) focuses on the contextual factors, and posits that processing of an ironic statement is facilitated if it echoes a previous contextual statement. For example, if John has mentioned that tomorrow the weather will be lovely for a picnic, Mary's text message in the next morning "What a lovely weather for a picnic!" when it is pouring rain echoes John's previous comment, and facilitates the recognition of it as ironic criticism.

The implicit display theory (Utsumi, 2000) emphasizes both contextual and phrase-related factors in irony comprehension. According to Utsumi (2000) irony is typically used when a speaker is disappointed and wishes to indirectly express a negative attitude, which violates the assumption that a speaker is sincere. This is what Utsumi calls prototypical irony, and suggests that if the discourse context is clearly ironic, and the statement has a high resemblance with prototypical irony, the intended meaning is comprehended quickly. Also, the graded salience view (Giora, 2003) emphasizes phraserelated features and posits that readers go with the most salient interpretation: if the ironic statement is highly familiar, it is more salient than literal meaning and accessed first, which speeds up processing. However, the defaultness hypothesis (Giora, Givoni, \& Fein, 2015) introduces an exception to this saliency-rule: sometimes non-salient interpretation is the default. For example, negative constructions 
(e.g., "He is not the smartest student in the class") are more quickly identified as irony, even though they are not familiar as irony.

However, comprehension of irony is likely to be influenced also by reader-related factors such as working memory capacity (WMC), which is expected to affect the efficiency of irony processing (Giora, 1999). Of the current theoretical views, only the parallel-constraint-satisfaction view (Katz \& Ferretti, 2001; Pexman, 2008) posits that various context-, phrase-, and reader-related factors influence the irony comprehension process. For example, processing and comprehending irony are easier if the contextual cues point towards an ironic interpretation, the statement is highly familiar as irony, and the reader is sensitive to irony (Ivanko, Pexman, \& Olineck, 2004) and has the necessary affectivecognitive abilities (e.g., Happé, 1993).

Eye tracking, which provides detailed information about the time-course of reading-related processes (see Rayner, 1998), helps in revealing the processes underlying irony comprehension. In the present study, we first report results of a meta-analysis of eye tracking studies on processing of ironic criticism, which (in certain cases) all theoretical views expect to be harder to comprehend and take longer to process than literal statements. Specifically, we examine whether ironic criticism causes localized and immediate slow-down of processing, reflected in the 'early measures' as longer fixation times during first-pass reading of the phrase, or whether the effects materialize in the 'late measures' as increased rereading of the phrase. The effects in the early measures would indicate that the meaning of ironic criticism is resolved on the spot, whereas effects in the late measures would suggest a slower process in which a reanalysis of the meaning is required before the phrase is integrated to the discourse representation. It is also important to consider the comprehension outcomes and therefore we include comparisons of comprehension of ironic criticism and literal phrases. We then report a systematic review of eye tracking studies that have examined the impact of various contextual, phrase-, and 
reader-related factors on irony processing. Based on this previous work, we will give recommendations for future research, and suggest that a novel theoretical view based on the predictive coding account of cognition (see Fabry, 2019) would be suitable for conceptualizing the irony effects.

\section{Method}

The eligibility criteria for the studies to be included in the meta-analysis and the review were: 1) study reported eye movement measures recorded during reading, 2) measures were reported for the ironic target phrase, and 3) experiment included a literal control condition. A search in the Web of Science was conducted using keywords "eye tracking AND sarcasm", "eye tracking AND irony", "eye movement AND sarcasm", and "eye movement AND irony". Google Scholar database was searched for articles with these keywords used in the title. Searches produced 39 hits, of which two were doctoral dissertations and four conference proceedings, which were left out from further analyses. The rest of the papers were screened for the eligibility criteria, resulting in ten papers to be included in the meta-analysis (see Table 1). The studies were coded for study design, number of participants and items, and language used in the study. For further information on the screening process, see Figure 1.

Means and standard deviations for eye movement measures and comprehension scores were extracted for ironic and literal conditions. In all studies the same target phrase was used in both conditions (e.g., "What a lovely day for a picnic!"), and context was used to bias it to either literal (it is a sunny day) or ironic (it is pouring rain) interpretation. Studies reported different measures, and we chose the ones that would capture early vs. late processing and that were reported in all studies. As an early processing measure, we used first pass fixation time, which is the summed duration of fixations made on the target phrase before moving away from the phrase (either forward or backwards). For late processing measures, we report two measures: look-back time and look-from time. Look-back time is 
the summed duration of fixations made to the target phrase from subsequent parts of text. Not all studies reported this measure, and from those we used total fixation time (the summed duration of all fixations made to the target phrase, including first-pass reading) as a proxy for look-back time. Another late processing measure reported is look-from time, which is the summed duration of look-back fixations that were initiated from the sentence. For studies that did not include this measure, we used regression path duration (the summed duration of fixations made on the target phrase and text segments preceding it in text before the reader moves on to the next text segment). The measures were extracted for the region in which irony becomes evident (target region or part of it), and the region immediately following the target region (spillover region). For the spillover region, only first-pass reading time was considered. As for the effects of ironic criticism on comprehension, we selected studies that had assessed how well the readers were able to comprehend the intended meaning of ironic and literal target phrases. Four of the studies used binary comprehension questions (e.g., "Did Mary think that it was nice day for a picnic.”), and one study (Olkoniemi, Strömberg, \& Kaakinen, 2019) used open comprehension questions (e.g., "In your opinion, what did Mary mean?").

If a paper reported more than one experiment with different samples, they were coded separately. If the study design included multiple conditions, we either selected the ironic criticism condition in which the target statement was not familiar as ironic criticism, or combined means and standard deviations across different conditions. From studies involving patient populations, only the control group was included. In all the studies included, the participants were native speakers of the language used in the experiment; studies did not provide information whether participants were monolingual or multilingual (see Tiv, Deodato, Rouillard, Wiebe, \& Titone, in press). Descriptive statistics were not reported in two papers, and they were requested directly from the authors. Unbiased estimate of Cohen's d (e.g., Cumming, 2012) was computed for each study using the standard deviation 
of literal condition as the unit of measure. A random effects model was used for computing the metaanalysis effect size separately for each eye movement measure. Forest plots and summary statistics were estimated using metaviz package (version 0.3.0, Kossmeier, Tran, \& Voracek, 2019) in R statistical software (version 3.6.2.). Data and analysis code are available at https://osf.io/w5fbs/.

\section{Results}

\section{Meta-analysis of processing and comprehension of ironic criticism}

The forest plots on the effect of irony on the different measures are presented in Figure 2. The meta-analysis showed that the irony effect in the first-pass reading time on target phrase was negligible, $d_{\mathrm{unb}}=0.11,95 \% \mathrm{CI}[-0.01,0.22]$. However, there was a clear irony effect in the look-back time to the target region, $d_{\mathrm{unb}}=0.22,95 \%$ CI $[0.11,0.33]$, indicating that readers spend longer time rereading ironic criticism than literal phrases. Moreover, there was an irony effect in the look-from time from the target region, $d_{\mathrm{unb}}=0.24,95 \% \mathrm{CI}[0.09,0.38]$, indicating that readers initiated more rereading of the text from ironic than literal phrases. The irony effect in the first-pass reading time of the spillover region was small, $d_{\mathrm{unb}}=0.10,95 \%$ CI [-0.02, 0.22]. As for comprehension (see Figure 3), the meta-analysis showed that ironic phrases are clearly harder to comprehend than literal phrases, $d_{\mathrm{unb}}=$ $1.03,95 \%$ CI $[-1.41,-0.65]$.

In sum, the results of the meta-analysis indicate that the effects of ironic criticism are seen in the late processing measures as increased rereading of the ironic phrase and returns to the preceding text context. Ironic phrases are also harder to understand than literal phrases. Next, we present a systematic review of different context-, phrase-, and reader-related factors that impact processing and comprehension of irony. The systematic review will elaborate on the ten studies included in the meta- 
analysis (see Table 1), with the goal of teasing apart the various factors that contribute to irony comprehension.

\section{Context-related factors influencing processing of irony}

The studies included in this review suggest that both the discourse context (Filik, Brightman, Gathercole, \& Leuthold, 2017; Turcan \& Filik, 2016, 2017) and the global study context influence processing of irony (Olkoniemi, Ranta, \& Kaakinen 2016; Olkoniemi, Johander, \& Kaakinen, 2019; Olkoniemi, Strömberg, et al., 2019). Turcan and Filik (2017) examined whether echoing a previous contextual utterance affects the reading times of ironic criticism versus literal target phrases. They found that echoic mention facilitated processing of ironic criticism, as indicated by shorter total reading times. However, reading times were overall higher in the spillover region for ironic phrases. Filik et al. (2017) manipulated the emotional context in which the phrase was presented. Participants read texts containing either literal or ironic criticism followed by the target phrase describing the victim of criticism being either hurt (Exp 1) or amused (Exp 2). The results showed that reading times were shorter for the hurtful response following ironic criticism when compared to literal criticism, but there was a slow-down in the spillover region. As for the amused response, reading times were shorter in the spillover region after ironic than literal criticism. Filik et al. suggested that a hurt response following ironic criticism is expected, but harder to integrate to the text representation, whereas an amused response is easier to integrate to the text representation. Even though these studies demonstrate that the discourse context is important in processing of irony, some studies have failed to demonstrate such effects. Turcan and Filik (2016) examined whether creating a context in which expectations about speaker's intent are explicit (vs. implicit) facilitates processing of ironic phrases, and found no effects. 
Olkoniemi and colleagues studied the role of global study context, and showed that the processing of ironic vs. literal phrases changes during an experimental session (Olkoniemi et al., 2016; Olkoniemi, Johander, et al., 2019; Olkoniemi, Strömberg, et al., 2019). The results suggest that when the reader repeatedly encounters ironic statements, there will be an expectation of forthcoming irony, which, in turn, reduces the level of confusion potentially caused by irony (see also Spotorno \& Noveck, 2014).

\section{Text-related factors influencing processing of irony}

Some previous studies have focused on the phrase-level factors such as familiarity (Filik, Leuthold, Wallington, \& Page, 2014; Turcan \& Filik, 2016, 2017) and the defaultness of interpretation (Filik, Howman, Ralph-Nearman, \& Giora, 2018). Findings related to familiarity of an ironic phrase are contradictory. Filik et al. (2014) examined processing of phrases that were familiar or unfamiliar in ironic contexts, and found that there was no processing time difference between ironic and literal phrases if the phrases were familiar as irony. Partly in line with this study, Turcan and Filik (2016) found that when the ironic phrase was familiar, regression path duration was similar for ironic and literal phrases. However, first-pass reading time and total reading time were longer for ironic vs. literal phrases. It should be noted that in the study, the target phrases were different in the familiar and unfamiliar conditions, which might have influenced the findings. Last, Turcan and Filik (2017) examined the effect of familiarity on processing of ironic criticism, and failed to find effects of familiarity on the processing of ironic vs. literal phrases.

As for the defaultness of interpretation, Filik and colleagues (2018) examined processing of negative (e.g., "He isn't the best lawyer") and affirmative (e.g., "He is the best lawyer") phrases, and found that negative phrases were more likely to be interpreted as ironic, whereas affirmative phrases 
were often perceived as literal (Filik et al., 2018). This difference was reflected in eye movements: For affirmative phrases fixation times were longer in ironic than in literal context, whereas for negative phrases the fixation times were longer in literal than in ironic context. These results indicate that the default interpretation - whether it is literal or ironic - has a processing advantage.

\section{Reader-related factors influencing processing of irony}

One of the reader-related factors that has attracted attention in previous studies is WMC. Results show that high WMC is related to an increase in the processing times of the ironic statements during the first-pass reading (Kaakinen, Olkoniemi, Kinnari, \& Hyönä, 2014; Olkoniemi et al., 2016; Olkoniemi, Johander, et al., 2019), whereas low WMC readers are more likely to look back to ironic statements from the other text parts (Olkoniemi et al., 2016; Olkoniemi, Johander, et al., 2019). These findings suggest that high WMC readers are able to start processing the intended meaning early, during the first-pass reading of the phrase. On the other hand, readers with low WMC are bound to use text as external memory and apply compensatory strategies, such as looking back in text. However, when the ironic statements are embedded in relatively short (3-4 sentences) paragraphs, the effect related to WMC seems to disappear (Olkoniemi, Strömberg, et al., 2019). This suggests that the effects of WMC only emerge when the text puts some strain on the reader's working memory.

Other reader-related factors associated with processing of irony are the ability to process emotional information and the tendency to use malicious humor (Filik et al., 2018; Olkoniemi et al., 2016; Olkoniemi, Johander, et al., 2019; Olkoniemi, Strömberg, et al., 2019). Studies by Olkoniemi and colleagues showed that a better ability to process emotional information was related to less processing time invested in the ironic statements (Olkoniemi et al., 2016; Olkoniemi, Johander, et al., 2019). In contrast, readers with a poorer ability to process emotional information showed increased 
processing times of paragraphs containing an ironic statement (Olkoniemi et al., 2016; Olkoniemi, Johander, et al., 2019; Olkoniemi, Strömberg, et al., 2019). Moreover, Filik and colleagues (2018, Exp 2) showed that readers' tendency to use malicious humour (e.g., use of sarcasm to insult others, and make fun of people in public) was related to shorter rereading of the ironic in comparison to literal statement (see also Ivanko et al., 2004).

Individuals with autism spectrum disorder (ASD) have challenges in social interaction leading to deficits in comprehending indirect language like irony (e.g., Happé, 1993). However, Au-Yeung, Kaakinen, Liversedge, and Benson (2015) found that participants with ASD performed at a comparable level to the typically developed (TD) group in irony comprehension. Both groups showed longer total fixation times and lower comprehension accuracy for ironic in comparison to literal statements in text, indicating that individuals with ASD are sensitive to irony. Barzy, Filik, Williams, and Ferguson (2020) examined how readers react to indications of character emotions after ironic criticisms, and showed that individuals with ASD have difficulties in comprehending how ironic criticism makes other people feel. In other words, while individuals with ASD do recognize the discrepancy between an ironic statement and the context in which it is presented and can understand its intended meaning, they have trouble in understanding its emotional consequences (Barzy et al., 2020).

\section{Conclusions and Future Directions}

Based on the meta-analysis, processing of ironic criticism is most notably characterized by increased rereading of the ironic phrases and returns to the preceding text context. These results suggest that a reanalysis of the meaning is required before the ironic phrase is integrated into the discourse representation. The systematic review revealed that this effect is modulated by different context-, 
phrase-, and reader-related factors. Before discussing the theoretical implications of these results, we will present some methodological considerations stemming from the present study.

First, many studies included in the present paper reported typical word-level fixation time measures (see Rayner, 1998), which are not optimal for describing the time-course of reading of phrases (Kaakinen, 2017). Irony is a property of a phrase, not of a single word, and the reader usually has to read the whole phrase in order to understand that it is ironic. For example, in the phrase "what a great day for a picnic", the word "great" defines the valence of the utterance, but the reader does not know what is great (or not) until they have read the whole phrase. Another problem is that word-level measures provide very little information about the time-course of processing of the phrase. Total fixation time and regression path duration do not reveal what happens during the first-pass reading of the phrase, whether it is read several times during first-pass reading, or whether there are later lookbacks to it. Phrase or sentence-level measures are thus better suited for revealing the time-course of processing of ironic vs. literal phrases (Kaakinen, 2017). Moreover, fixation time measures provide restricted information about the transitions between the different parts of text. An interesting approach for analyzing eye movements would be to examine readers' scan paths upon encountering ironic phrases. Scan path analysis has been successfully used to study the processing of garden path sentences and individual differences in how readers resolve them (e.g., von der Malsburg \& Vasishth, 2013).

Second, all the studies used textoids specifically written for the purpose of each study. While this allows maximal experimental control of factors that may influence eye movement measures (e.g., word length, frequency), they may not reflect real life communicative situations. As argued by Katz (2005): "The experimental stimuli should reflect real-life contextual constraints" (Katz, 2005, p. 188). We hope that in the future, more studies would use materials that are representative of real-life contexts, like ironic excerpts from literature or social media posts (see e.g. Mishra et al., 2016). 
Finally, comprehension was assessed only in few studies. However, outcome measures are crucial in understanding how the processing measures reflect the representations formed by the reader (Ferreira \& Yang, 2019). In other words, without comprehension measures we do not know if the eye movement patterns reflect successful comprehension of irony, or a comprehension failure.

Previous studies show that various context-, phrase-, and reader-related factors influence processing of ironic phrases. The only theoretical approach that currently acknowledges the role of all three types of factors is the parallel-constraint-satisfaction framework (Katz \& Ferretti, 2001; Pexman, 2008). However, there is a need for a better-specified theory that could be used to generate precise hypotheses about factors that impact (or do not impact) processing of irony. We suggest that applying the predictive coding theory to irony processing (Fabry, 2019) would be a promising approach. According to this view, irony comprehension can be described as a continuous attempt to minimize prediction error. As irony typically creates a contrast within the communicative context, it violates the predictions about what the character is likely to utter. However, the reader tries to minimize this prediction error by adjusting the priors for a literal (vs. ironic) interpretation. As shown by the current results, different context-, phrase-, and reader-related factors affect the magnitude of prediction error and the efficiency with which it is minimized. Developing a computational model based on predictive coding would allow testing the importance of specific parameters in irony processing. When combined with rigorous empirical work that utilizes time-sensitive methods such as eye tracking, we think that this approach would provide novel insight into the psychological underpinnings of irony comprehension. 


\section{References}

Attardo, S. (2000). Irony as relevant inappropriateness. Journal of Pragmatics, 32, $793-826$. doi:10.1016/S0378-2166(99)00070-3

Au-Yeung, S. K., Kaakinen, J. K., Liversedge, S. P., \& Benson, V. (2015). Processing of written irony in autism spectrum disorder: An eye-movement study. Autism Research, 8, 749-760. doi:10.1002/aur.1490

Barzy, M., Filik, R., Williams, D., \& Ferguson, H. J. (2020). Emotional processing of ironic versus literal criticism in autistic and nonautistic adults: Evidence from eye-tracking. Autism Research, 13, 563-578. doi:10.1002/aur.2272

Cumming, G. (2012). Understanding the new statistics: Effect sizes, confidence intervals, and metaanalysis. New York: Routledge.

Fabry, R. E. (2019). Getting it: A predictive processing approach to irony comprehension Synthese. doi:10.1007/s11229-019-02470-9

Ferreira, F., \& Yang, Z. (2019). The problem of comprehension in psycholinguistics. Discourse Processes, 56, 485-495. doi:10.1080/0163853X.2019.1591885

Filik, R., Brightman, E., Gathercole, C., \& Leuthold, H. (2017). The emotional impact of verbal irony: Eye-tracking evidence for a two-stage process. Journal of Memory and Language, 93, 193-202. doi:10.1016/j.jml.2016.09.006

Filik, R., Howman, H., Ralph-Nearman, C., \& Giora, R. (2018). The role of defaultness and personality factors in sarcasm interpretation: Evidence from eye-tracking during reading. Metaphor and Symbol, 33, 148-162. doi:10.1080/10926488.2018.1481258

Filik, R., Leuthold, H., Wallington, K., \& Page, J. (2014). Testing theories of irony processing using eye-tracking and ERPs. Journal of Experimental Psychology: Learning, Memory, and 
Cognition, 40, 811-828. doi:10.1037/a0035658

Filik, R., \& Moxey, L. M. (2010). The on-line processing of written irony. Cognition, 116, 421-436. doi:10.1016/j.cognition.2010.06.005.

Gibbs, R. W. (1994). The poetics of mind: Figurative thought, language, and understanding. Cambridge: Cambridge University Press.

Giora, R. (1999). On the priority of salient meanings: Studies of literal and figurative language. Journal of Pragmatics, 31, 919-929. doi:10.1016/S0378-2166(98)00100-3

Giora, R. (2003). On our mind: Salience, context, and figurative language. New York: Oxford University Press.

Giora, R., Givoni, S., \& Fein, O. (2015). Defaultness reigns: The case of sarcasm. Metaphor and Symbol, 30, 290-313. doi:10.1080/10926488.2015.1074804

Grice, H. (1975). Logic and conversation. In P. Cole \& J. Morgan (Eds.), Syntax and semantics 3: Speech acts (pp. 41-58). New York: Academic Press.

Happé, F. G. E. (1993). Communicative competence and theory of mind in autism: A test of relevance theory. Cognition, 48, 101-119. doi:10.1016/0010-0277(93)90026-R

Hancock, J. T. (2004). Verbal irony use in face-to-face and computer-mediated conversations. Journal of Language and Social Psychology, 23, 447-463. doi:10.1177/0261927X04269587

Ivanko, S. L., Pexman, P. M., \& Olineck, K. M. (2004). How sarcastic are you?: Individual differences and verbal irony. Journal of Language and Social Psychology, 23, 244-271. doi:10.1177/0261927X04266809

Kaakinen, J. K. (2017). On-line measures of text processing. In M. F. Schober, D. N. Rapp, \& M. A. Britt (Eds.), The Routledge handbook of discourse processes: Second edition (pp. 125-130). New York: Routledge. 
Kaakinen, J. K., Olkoniemi, H., Kinnari, T., \& Hyönä, J. (2014). Processing of written irony: An eye movement study. Discourse Processes, 51, 287-311. doi: 10.1080/0163853X.2013.870024

Katz, A. N. (2005). Discourse and sociocultural factors in understanding nonliteral language. In A. N. Katz (Ed.) Figurative language comprehension: Social and cultural influences (pp. 183-207). New Jersey: Lawrence Erlbaum Associates Publishers.

Katz, A. N., Blasko, D. G., \& Kazmerski, V. A. (2004). Saying what you don’t mean: Social influences on sarcastic language processing. Current Directions in Psychological Science, 13, 186-189. doi:10.1111/j.0963-7214.2004.00304.X

Katz, A. N., \& Ferretti, T. R. (2001). Moment-by-moment reading of proverbs in literal and nonliteral contexts. Metaphor and Symbol, 16, 193-221. doi:10.1080/10926488.2001.9678895

Kossmeier, M., Tran, U. S., \& Voracek, M. (2020). metaviz: Forest plots, funnel plots, and visual funnel plot inference for meta-analysis (R package version 0.30).

Kreuz, R. J., \& Link, K. E., (2002). Asymmetries in the use of verbal irony. Journal of Language and Social Psychology, 21, 127-143. doi: 10.1177/02627X02021002002

Mishra, A., Kanojia, D., \& Bhattacharyya, P. (2016). Predicting readers' sarcasm understandability by modeling gaze behavior. Proceedings of the Thirtieth AAAI Conference on Artificial Intelligence, 5, 3747-3753.

Olkoniemi, H., Johander, E., \& Kaakinen, J. K. (2019). The role of look-backs in the processing of written sarcasm. Memory \& Cognition, 47, 87-105. doi:10.3758/s13421-018-0852-2

Olkoniemi, H., \& Kaakinen, J. K. (2020). Processing of irony in text: A systematic review of eye tracking studies [Data set]. https://osf.io/w5fbs/

Olkoniemi, H., Ranta, H., \& Kaakinen, J. K. (2016). Individual differences in the processing of written sarcasm and metaphor: Evidence from eye movements. Journal of Experimental Psychology: Learning, Memory, and Cognition, 42, 433-450. doi:10.1037/xlm0000176 
Olkoniemi, H., Strömberg, V., \& Kaakinen, J. K. (2019). The ability to recognise emotions predicts the time-course of sarcasm processing: Evidence from eye movements. Quarterly Journal of Experimental Psychology, 72, 1212-1223. doi:10.1177/1747021818807864

Pexman, P. M. (2008). It's fascinating research: The cognition of verbal irony. Current Directions in Psychological Science, 17, 286-290. doi:10.1111/j.1467-8721.2008.00591.x

Rayner, K. (1998) Eye movements in reading and information processing: 20 years of research. Psychological Bulletin, 124, 372-422.

Sperber, D., \& Wilson, D. (1981). On verbal irony. In P. Cole (Ed.), Radical Pragmatics (pp. 295318). New York: Academic Press.

Spotorno, N., \& Noveck, I. A. (2014). When is irony effortful? Journal of Experimental Psychology: General, 143, 1649-1665. doi:10.1037/a0036630

Tiv, M., Deodato, F., Rouillard, V., Wiebe, S., \& Titone, D. (in press). Second language experience impacts first language irony comprehension among bilingual adults. Canadian Journal of Experimental Psychology.

Toplak, M., \& Katz, A. N. (2000). On the uses of sarcastic irony. Journal of Pragmatics, 32, 14671488. doi: $\underline{10.1016 / \mathrm{S} 0378-2166(99) 00101-0}$

Turcan, A., \& Filik, R. (2016). An eye-tracking investigation of written sarcasm comprehension: The role of familiarity and context. Journal of Experimental Psychology: Learning, Memory, and Cognition, 42, 1867-1893. doi:10.1037/xlm0000285

Turcan, A., \& Filik, R. (2017). Investigating sarcasm comprehension using eye-tracking during reading: What are the roles of literality, familiarity, and echoic mention? In A. Athanasiadou, \& H. L. Colston (Eds.) Irony in language use and communication (pp. 55-276). Amsterdam: John Benjamins Publishing Company. doi:10.1075/ftl.1.13tuc

Utsumi, A. (2000). Verbal irony as implicit display of ironic environment: Distinguishing ironic 
utterances from nonirony. Journal of Pragmatics, 32, 1777-1806.

doi:10.1016/S0378-2166(99)00116-2

von der Malsburg, T., \& Vasishth, S. (2013). Scanpaths reveal syntactic underspecification and reanalysis strategies. Language and Cognitive Processes, 28, 1545-1578.

doi:10.1080/01690965.2012.728232 
Table 1

Studies included in the meta-analysis and the review

Included in meta- Comprehension

\begin{tabular}{llllll}
\multicolumn{1}{c}{ Study } & Language & $N$ & $N$ (items) & analysis & measured \\
\hline Au-Yeung et al. (2015) & English & 19 & 36 & Yes & Yes \\
Barzy et al. (2020) & English & 24 & 56 & No & No \\
Filik \& Moxey (2010) & English & 48 & 48 & Yes & No \\
Filik et al. (2017) Exp 1 & English & 28 & 32 & No & No \\
Filik et al. (2017) Exp 2 & English & 28 & 32 & No & No \\
Filik et al. (2018) & English & 40 & 24 & Yes & No \\
Filik et al. (2014) & English & 44 & 44 & Yes & No \\
Kaakinen et al. (2014) Exp 1 & Finnish & 51 & 24 & Yes & Yes \\
Kaakinen et al. (2014) Exp 2 & Finnish & 60 & 24 & Yes & Yes \\
Olkoniemi, Johander et al. (2019) & Finnish & 62 & 28 & Yes & Yes \\
Olkoniemi et al. (2016) & Finnish & 60 & 20 & Yes & Yes \\
Olkoniemi, Strömberg et al. (2019) & Finnish & 60 & 40 & Yes & Yes \\
Turcan \& Filik (2016) Exp 1 & English & 32 & 24 & Yes & No \\
Turcan \& Filik (2016) Exp 2 & English & 64 & 48 & Yes & No \\
Turcan \& Filik (2017) & English & 64 & 48 & Yes & No \\
\hline Note. Onl typically deves &
\end{tabular}

Note. Only typically developed individuals were included from the Au-Yeung et al. (2015) study. 


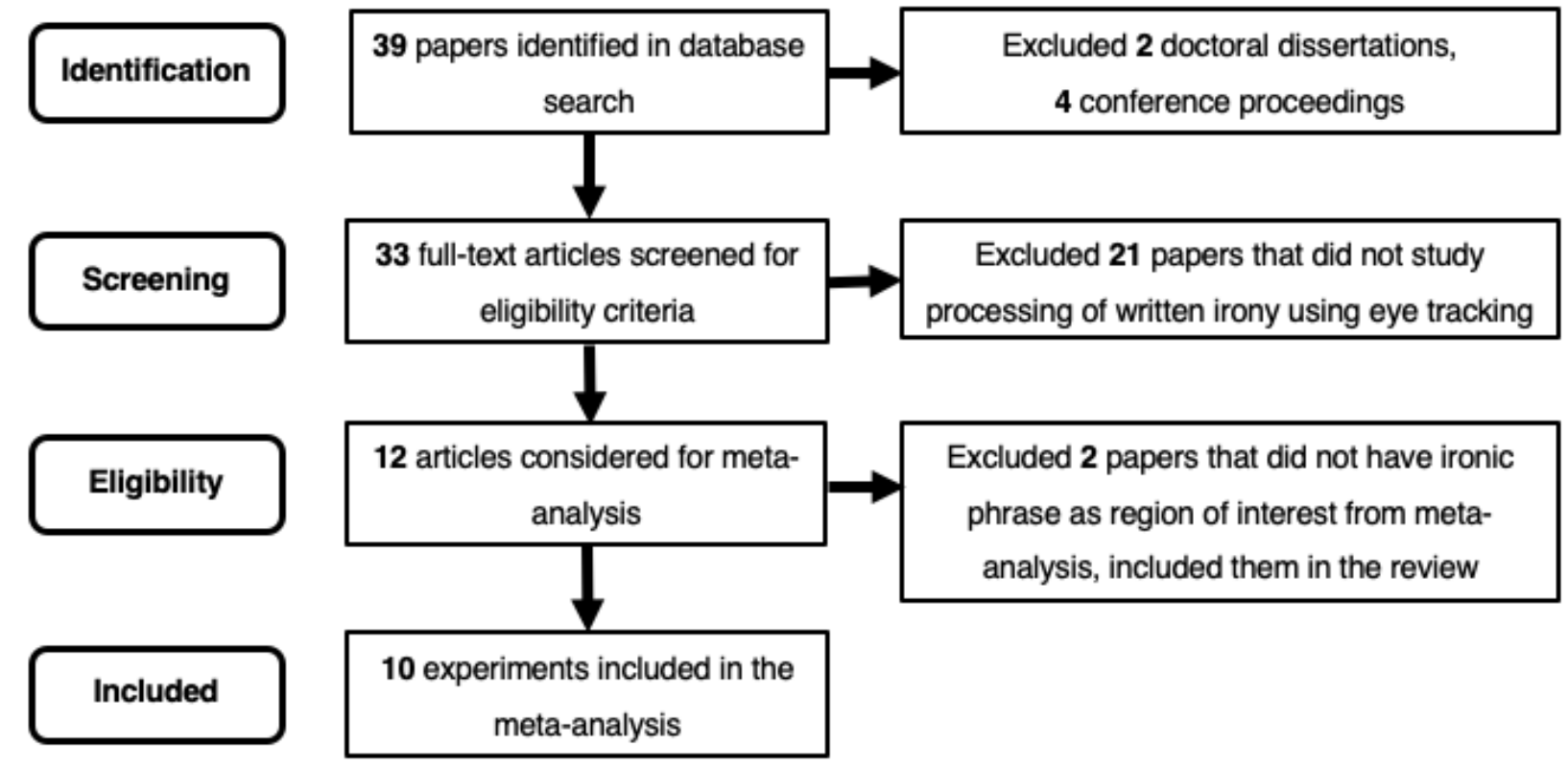

Figure 1. Flow chart for the screening and inclusion of studies. 
A

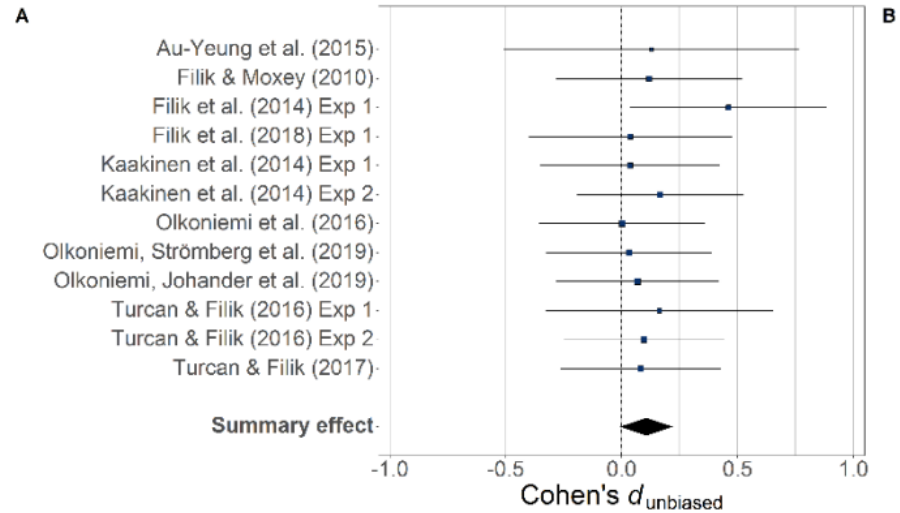

c

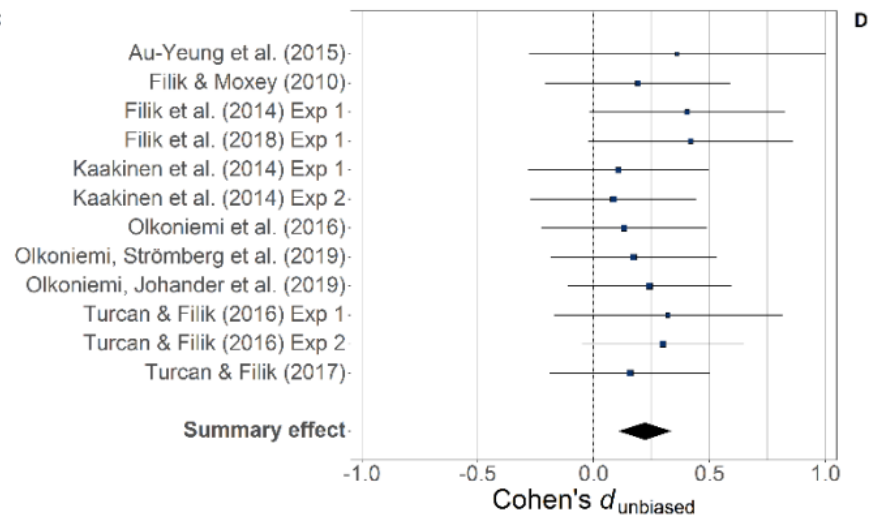

B

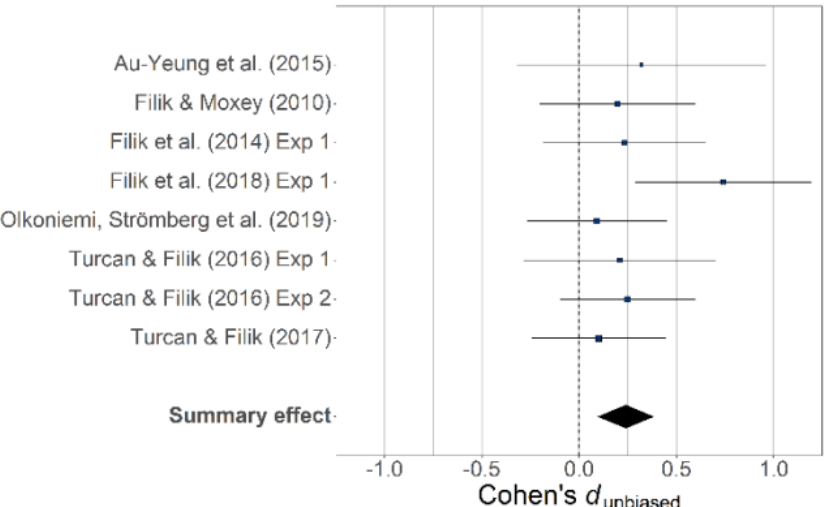

D

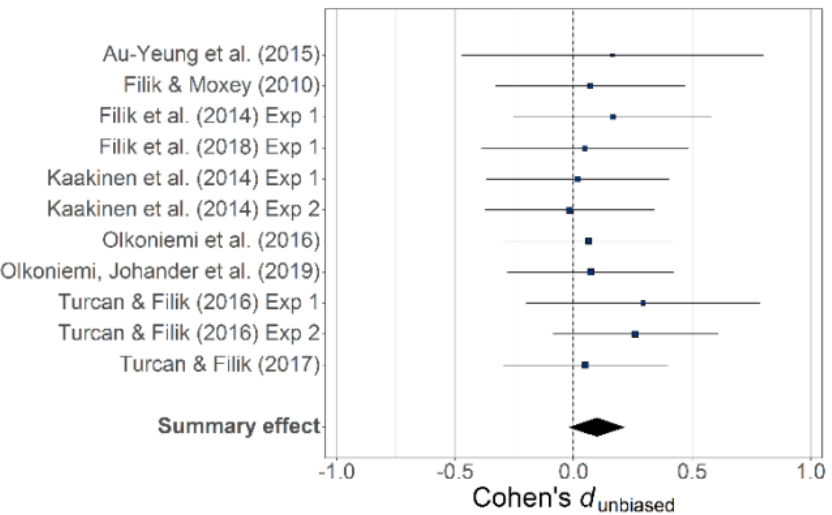

Figure 2. Forest plots on effect of irony (in comparison to literal) of the ten studies included in the meta-analysis on different eye movement measures. X-axis represents unbiased Cohen's d. Error bars represent $95 \%$ confidence intervals. A) Represents first-pass reading time on the target phrase. B) Represents look-from time from the target phrase. C) Represent look-back time to target phrase. D) Represents first-pass reading time on the spillover region. 


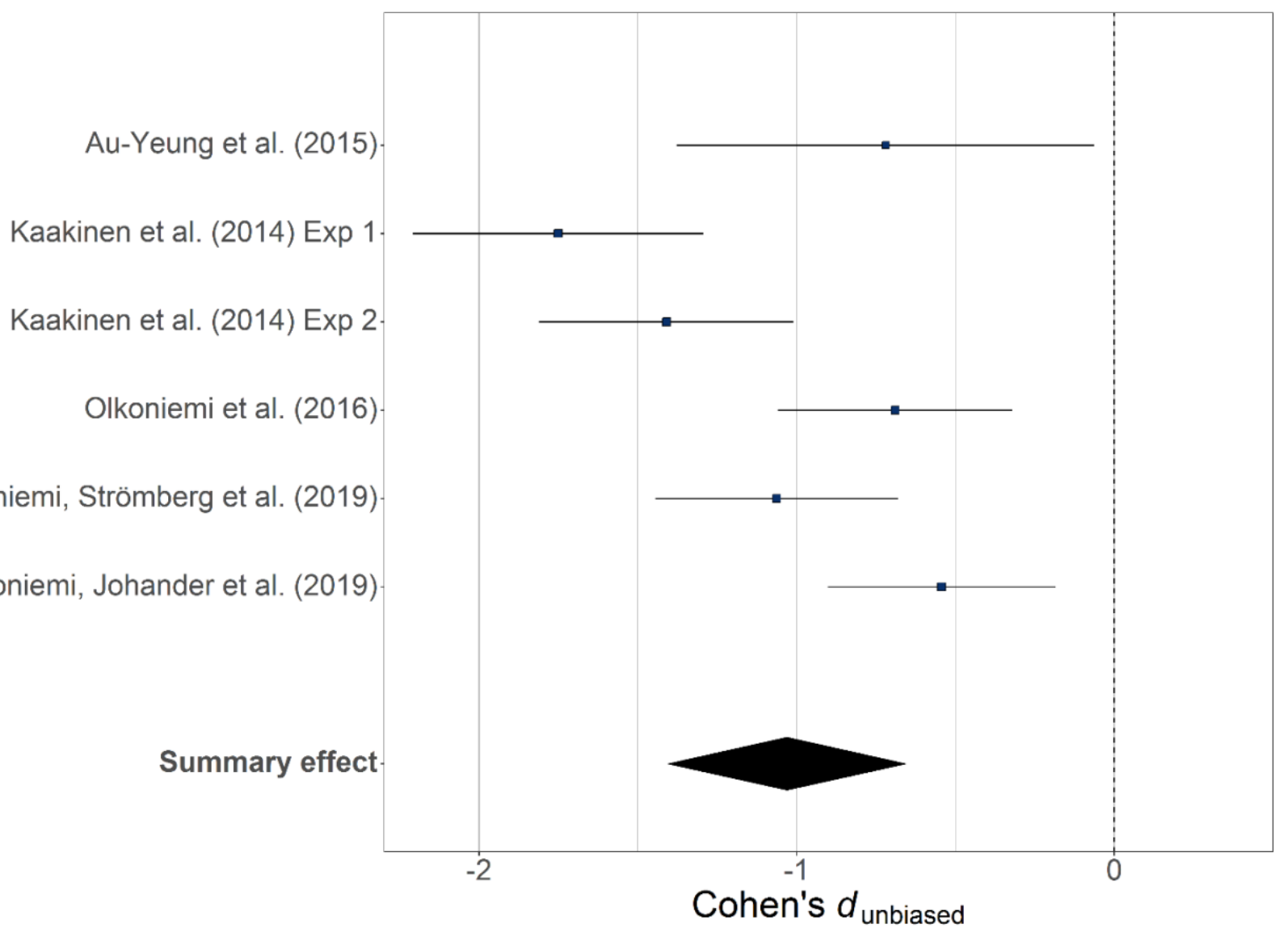

Figure 3. Forest plots on irony comprehension across the five studies. X-axis represents unbiased Cohen's d. Error bars represent 95\% confidence intervals. 